\title{
Obsessive-compulsive disorder presenting as chronic dysphagia-a case report
}

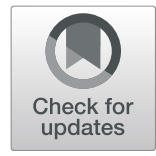

Sadia Sultan (D)

\begin{abstract}
Background: Medically unexplained oropharyngeal dysphagia (MUNOD) is a rare condition. It presents without demonstrable abnormalities in the anatomy of the upper aero-digestive tract or swallowing physiology. The aim of this case report is to discuss the unique presentation of OCD as chronic dysphagia.

Case presentation: A 65-year-old woman was admitted with dysphagia and weight loss in surgical department with history of undergoing repeated upper Gl endoscopies and laryngoscopies. The patient was finally diagnosed to be suffering from chronic obsessive-compulsive disorder with poor insight and comorbid depression after 3 years of suffering and moving from hospital to hospital. The atypical presentation and poor insight were the major reasons for diagnostic difficulty in this particular case. The patient was given $60 \mathrm{mg}$ fluoxetine and $2 \mathrm{mg}$ risperidone which improved the patient's symptoms by $40 \%$.

Conclusion: This case explains the possibility of obsessive-compulsive disorder in patients presenting with unexplained somatic symptoms. It also demonstrated the importance of a timely psychiatric diagnosis and treatment and highlights the importance about awareness of psychiatric illness in doctor community which can reduce patient suffering and unnecessary invasive procedures.
\end{abstract}

Keywords: Medically unexplained oropharyngeal dysphagia, Obsessive-compulsive disorder, Psychogenic dysphagia

\section{Background}

Patients with swallowing problems commonly end up at otorhinolaryngology or gastroenterology outpatient clinic. Head and neck cancer, progressive neurological disorders or stroke are among the list of causes leading to oropharyngeal dysphagia (OD) [1-3]. Medically unexplained oropharyngeal dysphagia (MUNOD) is described as dysphagia occurring without a demonstrable abnormality in the anatomy or physiology of upper aerodigestive tract [4]. Functional dysphagia, swallowing phobia, psychogenic dysphagia or phagophobia are the various names described in the literature for this particular condition [4]. Physical complaints or symptoms which affect the normal functioning of the body without underlying medical condition or structural disease is described as functional somatic disorder [5]. "MUNOD"

Correspondence: drsaddy2003@yahoo.com

Department of Laboratory Medicine, College of Applied Medical Sciences, Umm Al-Qura University, Mecca, Saudi Arabia may be a symptom within other psychiatric conditions such as obsessive-compulsive disorder, panic disorder, post-traumatic stress disorder and social phobia [6]. "Obsessive-compulsive disorder (OCD) is characterized by repetitive, intrusive thoughts and images and/or by repetitive, ritualistic physical or mental acts performed to reduce the attendant anxiety" [7]. Intrusive thoughts that allude to processes like awareness of breathing, blinking, swallowing, body positioning and physical sensations are described as somatic obsessions (sensorimotor or somatoform obsessions). Many OCD symptoms may shift over time along a range from "definitely logical" to "extremely irrational thoughts and behaviour". The diagnosis of OCD may be difficult in patients who surrender the struggle against their symptoms, which seem to change from unwanted and distressing intrusion to psychotic delusion [8]. The aim of this case report is to discuss the atypical presentation leading to diagnostic difficulty and also highlights the 
importance of awareness of psychiatric illness in doctor community.

\section{Case presentation}

A 60-year-old female, referred from surgical inpatient department for evaluation by a psychiatrist, was married and uneducated hailing from rural background presenting with inability to swallow and chronic weight loss since the last 3 years. This female has developed nonspecific throat symptoms with inability to swallow and feeling of lump in the throat which gradually prevented her from eating to the extent that she became cachexic, lost $10 \mathrm{~kg}$ weight and was hospitalized. Neither the patient nor the informant could recall any stressor preceding the symptoms. During the past 3 years, she was treated by various GPs, ENT surgeons and gastroenterologists and underwent multiple endoscopies and laryngoscopies with normal results. She was initially referred to the surgical outpatient department by a duty medical officer from the emergency department for evaluation of chronic dysphagia, undiagnosed since the last 3 years and was subsequently sent to the psychiatry department for evaluation.

Rapport was established with difficulty since she was hardly talking and appeared very weak and fragile; her only complaint was inability to swallow due to a lump in the throat since the last 3 years. Collateral information revealed that the patient has not been taking meals due to inability to swallow, lost $10 \mathrm{~kg}$ of weight, remains alone and aloof, often cries, does not sleep and expresses hopelessness and death wish. However, these depressive symptoms started only 6 months ago. On further enquiry about the past and asking questions pertaining to symptomology of OCD, it was revealed that she washed hands so often that her hands got excoriated, spent more time than required for the daily house hold chores and kept organizing things to the extent of measuring things in order to obtain perfect positioning. The informant being a daughter remembers this behaviour lasting since past 30 years with waxing and waning in the severity of symptoms. However, these symptoms are no longer present. Since last 3 years, she started complaining of the inability to swallow and checking behaviour was noticed. Initially, she was able to swallow with spilling and spitting out and frequently checked in the mirror for any deformity in the neck. Since the last 1 month, she is on fluids which also spill out very often only while drinking fluids, but drooling of saliva is otherwise not reported. Although drooling, coughing, choking, nasal regurgitation and any change in speech quality was not reported, she no longer checks or complains about anything. Patient is a known diabetic on metformin $1000 \mathrm{mg}$ with good glycemic control. No history of any other medical illness. No history of psychiatric illness in the family.
Any anankastic trait in the premorbid history could not be elicited due to lack of information. Physical examination revealed no significant findings. Motor, sensory and cranial nerve examination was normal. On mental status examination, she appeared dull, weak and ill groomed; rapport was established with difficulty. Although she expressed hopelessness regarding the cure of her illness, suicidal ideation was not reported; her speech was scant with low tone and affect was blunted with reduced reactivity. The belief of lump in throat and swallowing difficulty was ego-syntonic and at a delusional level with poor insight into the illness. She complained of repeated intrusive thoughts of being strangulated and choked. No other thought and perceptual abnormality was elicited during history taking or mental status examination. The patient cognitive function was intact. Routine investigations were normal including thyroid function test and serum vitamin B12. Neither CT nor MRI brain was done depending on lack of any neurologic deficit on physical examination and financial constraints of patient. The diagnosis of OCD with poor insight and comorbid depression was considered. The Yale Brown Obsessive-Compulsive Scale (Y-BOC Scale) revealed moderate scores $($ score $=20)$. Overvalued Idea Scale (OVIS) exhibited higher scores (score $=7$ ) suggestive of poor insight. Becks Depressive Inventory revealed moderately increased (score $=24)$. Score on the Positive and Negative Syndrome Scale (PANSS) was not suggestive of psychosis although the somatic concerns were at a delusional level. Patient was started on $20 \mathrm{mg}$ fluoxetine which was gradually increased to $60 \mathrm{mg}$ over a period of 4 weeks. At week three, $2 \mathrm{mg}$ risperidone was added. The patient reported appreciable improvement in symptoms in terms of ability to swallow (30-40\% as reported by the patient and Y-BOC Scale score). Her depressive symptoms also improved which was revealed in significant reduction in scores of Becks Depression Inventory. Tolerability and adherence to the treatment was good as reported by informants. She was advised sessions for exposure response prevention therapy at week 5 for which she showed low compliance and lost follow-up.

\section{Discussion}

The current case at the time of presentation seemed like a severe depression with psychosis. On first encounter with the patient, she appeared depressed and was only complaining of total inability to swallow with conviction, which was confused with nihilistic delusions. The fact that belief of inability to swallow was followed by depressive symptoms redirected the clinical suspicion towards somatic obsession and questioning pertaining to symptomology of OCD revealed history of multiple waxing and waning obsessions and compulsions in the past further supporting the diagnosis of OCD with poor 
insight and comorbid depression. The rejection of the fact that the obsessions and compulsions are meaningless and excessive by the patient is defined as poor insight, [9] which could have been the reason for missing the diagnosis of OCD in this particular case. The Diagnostic and Statistical Manual of Mental Disorders 5th edition [10] included two specifiers for OCD diagnosis: the presence of tics and, precisely, the level of insight, which may be classified in "good or fair" insight, "poor insight" and "absent" insight/delusional beliefs. Poor insight was indicated to be prevalent in $15-36 \%$ of OCD patients [9]. It has been demonstrated that poor insight is related to symptom severity, early onset of the illness, longer duration of the illness, compulsive hoarding, [11] somatic obsessions [12] and accompanying depressive symptom severity [13]. Major depression is the most common psychiatric comorbidity in OCD patients with poor insight [13]. Nonetheless, all the mentioned factors associated with poor insight OCD were found to be present in this particular case.

\section{Conclusion}

This case report specifically explores the issue of OCD not being diagnosed for almost 30 years and also highlights the difficulty in diagnosing OCD with poor insight. It also calls attention to the importance about awareness of psychiatric illness in doctor community which can reduce patient suffering and unnecessary invasive procedures.

\section{Abbreviations \\ MUNOD: Medically unexplained oropharyngeal dysphagia; OCD: Obsessive- compulsive disorder; OD: Oropharyngeal dysphagia; OVIS: Overvalued Idea Scale; PANSS: Positive and Negative Syndrome Scale; Y-BOC Scale: Yale Brown Obsessive-Compulsive Scale}

\section{Acknowledgements}

This research would not have been possible without the exceptional support of our Dean, Dr. Aiman Alsaegh, and Head of the Department, Dr. Ahmed H. Qassem, of Laboratory Medicine, Faculty of Applied Medical Sciences, Umm Al-Qura University.

\section{Author's contributions}

Dr. SS substantially contributed to conception, design, acquisition, interpretation of data, drafted and revised the work, finally approved the version to be published and was responsible for agreement to be accountable for all aspects of the work in ensuring that questions related to the accuracy or integrity of any part of the work are appropriately investigated and resolved. The author has read and approved the manuscript.

\section{Funding}

This research did not receive any grant from funding agencies in the public, commercial or not-for-profit sectors.

\section{Availability of data and materials}

Data sharing is not applicable to this article as no new data were created or analysed in this study.

Ethics approval and consent to participate

Not applicable

\section{Consent for publication}

Oral informed consent was obtained from the patient considering the fact that patient was illiterate. Moreover, no personal details were revealed in the case report.

\section{Competing interests}

The author declares she has no conflicts of interest.

Received: 8 October 2020 Accepted: 24 November 2020

Published online: 03 December 2020

\section{References}

1. Cichero JA (2006) Dysphagia foundation, theory and practice. Wiley, Chichester

2. Cook IJ, Kahrilas PJ (1999) AGA technical review on management of oropharyngeal dysphagia. Gastroenterology 116(2):455-478. https://doi.org/ 10.1016/50016-5085(99)7

3. Logemann J (1997) Evaluation and treatment of swallowing disorders. Austin: PRO-ED

4. Baijens LW, Koetsenruijter K, Pilz W (2013) Diagnosis and treatment of phagophobia: a review. Dysphagia 28(2):260-270. https://doi.org/10.1007/ s00455-013-9454-0

5. Fink P, Schroder A (2010) One single diagnosis, bodily distress syndrome, succeeded to capture 10 diagnostic categories of functional somatic syndromes and somatoform disorders. J Psychosom Res 68(5):415-426 doi: 10.1016/j.jpsychores.2010.02.004. 0

6. RJ MN (1994) Choking phobia: a review of the literature. Compr Psychiatry 35(1):83-89 doi: 10.1016/0010-440X(94)90174-0. 2

7. Kessler RC, Berglund P, Demler O, Jin R, Merikangas KR, Walters EE (2005) Lifetime prevalence and age-of-onset distributions of DSM-IV disorders in the National Comorbidity Survey Replication. Arch Gen Psychiatry 62:593602

8. Matsunaga H, Kiriike N, Matsui T, Iwasaki Y, Koshimune K, Ohya K et al (2002) Obsessive-compulsive disorder with poor insight. Comprehensive Psychiatry 42(2):150-157

9. Carmin C, Wiegartz PS, Wu K. Obsessive - compulsive disorder with poor insight. In Abramowitz JS, McKay D, Taylor S, editors. (2008). Clinical handbook of obsessive - compulsive disorder and related problems. Baltimore: The John Hopkins University Press: 109-125.

10. American Psychiatric Association (2013) Diagnostic and statistical manual of mental disorders, 5th edn. American Psychiatric Publishing, Arlington. https://doi.org/10.1176/appi.books.9780890425596

11. Ravi Kishore V, Samar R, Janardhan Reddy YC, Chandrasekhar CR, Thennarasu K (2004) Clinical characteristics and treatment in poor and good insight obsessive - compulsive disorder. Eur Psychiatry 19:202-208

12. Lochner C (2005) Cluster analysis of obsessive-compulsive spectrum disorders in patients with obsessive compulsive disorder: Clinical and genetic correlates. Compr Psychiatry. 46:14-19

13. Turksoy N, Tukel R, Ozdemir O, Karali A (2002) Comparison of clinical characteristics in good and poor insight obsessive - compulsive disorder. J Anxiety Disord 16:413-423

\section{Publisher's Note}

Springer Nature remains neutral with regard to jurisdictional claims in published maps and institutional affiliations. 